\title{
SISTEMATIZAR PROCEDIMENTOS OPERACIONAIS PARA A IMPLANTAÇÃO DE UM SISTEMA DE GESTÃO DAS OPERAÇÕES EM ORGANIZAÇÕES DA INDÚSTRIA DA SAÚDE
}

\section{OPERATIONAL PROCEDURES SYSTEMATIZATION FOR IMPLEMENTING AN OPERATIONS MANAGEMENT SYSTEM FOR ORGANIZATIONS IN THE HEALTH INDUSTRY}

\author{
Eduardo Gadotti Martins ${ }^{1}$; Edson Pinheiro de Lima ${ }^{2}$; Sergio Eduardo Gouvea da Costa ${ }^{3}$ \\ ${ }^{1}$ Pontifícia Universidade Católica do Paraná - PUCPR - Curitiba - Brasil \\ eduardo@gadotticar.com.br \\ ${ }^{2}$ Pontifícia Universidade Católica do Paraná - PUCPR - Curitiba - Brasil \\ Universidade Tecnológica Federal do Paraná - UTFPR - Curitiba - Brasil \\ e.pinheiro@pucpr.br, \\ ${ }^{3}$ Pontifícia Universidade Católica do Paraná - PUCPR - Curitiba - Brasil \\ Universidade Tecnológica Federal do Paraná - UTFPR - Curitiba - Brasil \\ s.gouvea@pucpr.br
}

\begin{abstract}
Resumo
Os regulamentos técnicos são estabelecidos pelos governos através de um agente específico, visando garantir a segurança e a saúde dos usuários de produtos para uso médico. A adaptação dos equipamentos e de seus fabricantes às normas e regulamentos técnicos estimula melhorias qualitativas nos produtos e processos de fabricação. $O$ objetivo desta pesquisa é de sistematizar um processo já proposto para a implantação de um sistema de gestão das operações em organizações da indústria da saúde orientada pelas normas ISO 13485 e da RDC $N^{o} .59$ desenvolvendo procedimentos operacionais para a aplicação prática. Tal implantação atende as demandas dos órgãos fiscalizadores e integra o sistema aos objetivos estratégicos da empresa. O método é desenvolvido utilizando-se a abordagem por processos (Cambridge approach), constituído por um conjunto de ações operacionais com seus respectivos indicadores, auxilie numa sequência de atividades que sejam planejadas $e$ organizadas para atender todas as exigências da norma e requisitos regulatórios. Para isto foram realizadas entrevistas com especialistas acadêmicos e não acadêmicos colhendo observações de modo a identificar possibilidades de aplicação com base na racionalidade e críticas dos pares. Não se pode ainda concluir que a metodologia proposta fornece os procedimentos práticos necessários para sua aplicação, mas já se observou que a metodologia se aperfeiçoou de modo a possibilitar a análise de sua aplicação em futuro estudo de caso.
\end{abstract}

Palavras-chave: Resolução RDC N . 59; Abordagem por Processos; ISO 13485; Requisitos Regulatórios; Gestão das Operações. 


\section{Introdução}

Um significativo custo incorrido por empresas da área de saúde é a certificação de produtos, cujo processo demanda, além de recursos financeiros, tempo. Os regulamentos técnicos são estabelecidos pelos governos através de um agente específico, visando garantir a segurança e a saúde dos usuários de produtos para uso médico. Os produtos que não estiverem de acordo com tais regulamentos têm sua comercialização proibida. Essas exigências são aplicadas igualmente aos produtos fabricados nacionalmente e aos importados.

Ao mesmo tempo em que visa proteger a integridade física dos usuários, a exigência de certificação pode criar um ciclo virtuoso entre os sistemas regulador e produtivo. A adaptação dos equipamentos e de seus fabricantes aos regulamentos técnicos estimula melhorias qualitativas nos produtos e processos de fabricação. Sendo assim, este setor passa a observar mais cuidadosamente a importância de se atender às exigências da legislação, tanto nos países desenvolvidos como nos países em desenvolvimento (IPPOLITI, FALAVIGNA, 2012).

No mercado global de hoje, muitas organizações estão utilizando a norma ISO 13485 como uma plataforma para construir seus sistemas de gerência do negócio na área de saúde. $\mathrm{O}$ objetivo da ISO 13485 é harmonizar os requisitos regulatórios de dispositivos médicos e sistema de gerência de qualidade. A ISO 13485:2003 referencia a norma ISO 14971 de Gerência de Riscos para Dispositivos Médicos, mas que pode ser utilizada apenas quando for uma exigência; mesmo não sendo requisito para alguns países, recomenda-se fortemente que as organizações considerem sua aplicabilidade ou relevância antes de desenvolverem algumas de suas próprias ferramentas da avaliação de risco (TROSCHINETZ, 2010).

No Brasil os equipamentos biomédicos seguem um regulamento que conforme sua classificação quanto ao potencial de risco à saúde de seus usuários (pacientes e/ou operadores), necessitam de registro da Agência Nacional de Vigilância Sanitária (ANVISA) para que os fabricantes os lancem no mercado consumidor. Além disto, sua indústria deve estar inserida dentro das boas práticas de fabricação obedecendo aos critérios estabelecidos pela ANVISA através da Resolução RDC (Resolução da Diretoria Colegiada) No. 59 de 27 de junho de 2000 (o título original da RDC N 59/2000 é "Boas Práticas de Fabricação de Produtos Médico - BPF”). A Resolução RDC No 59/2000 de 27 de junho 2000 é um conjunto de requisitos exigidos pela ANVISA para que os fabricantes e distribuidores de produtos médicos e odontológicos adotem em suas empresas e é similar ao Good Manufacturing Practice Regulations (GMP) do órgão americano Food and Drug Administration (FDA). 
No entanto, a dificuldade na implantação destas normas - ISO 13485:2003 e a RDC N 59/2000 - é a mesma encontrada em outros sistemas de qualidade, a exemplo da ISO 9001. Estas normas, assim como todas aquelas relacionadas aos Sistemas de Qualidade, dizem apenas ao que deve ser feito e não a como fazer. Além disto, a ANVISA enfrenta um agravante, qual seja a falta de padronização e preparo de alguns auditores (SO SERVIÇOS ORGANIZACIONAIS, 2011), ou mesmo a própria discordância de interpretações pelos mesmos profissionais, conforme observado por Sharp (1983).

Em resumo, por ser a RDC no 59 compulsória, enquanto a ISO 13485 uma exigência para exportação em muitos países, aliado às dificuldades acima apontadas e da própria dificuldade das empresas em compilarem referidas normas num pilar a possibilitar o desenvolvimento de um Sistema de Gestão Empresarial de forma a ganharem uma visão sistêmica do negócio e de toda a cadeia de relações de autoridade, responsabilidades, objetivos estratégicos, resultados esperados e alcançados na qual estão inseridas, o cumprimento de tais normativas tem sido encarado pela maioria, equivocadamente, apenas como uma exigência cartorial (VAZ et al, 2012).

Percebe-se, portanto, que a integração do Sistema de Gestão da Qualidade (SGQ) juntamente com os objetivos estratégicos do negócio, é em verdade um desafio para grande parte das empresas, que o vêem como responsabilidade única e exclusiva do departamento da qualidade Trata-se, no entanto, de uma visão míope e de pouco futuro. Recentemente, tem sido desenvolvidos modelos para a gestão integrada das operações que reforçam a orientação para resultados e a orientação estratégica para entender o conjunto total da cadeia de valor (NUDURUPATI et al, 2011; GIMBERT et al, 2010; GARCIA e COSTA, 2008; FRANCOSANTOS, 2007; HENRY, 2006).

O objetivo final desta pesquisa é propor um processo para a implantação de um sistema de gestão das operações em organizações da indústria da saúde orientada pelas normas ISO 13485 e da RDC Nº 59. Tal implantação atende às demandas dos órgãos fiscalizadores e integra o sistema aos objetivos estratégicos da empresa. O método é desenvolvido utilizando-se a abordagem por processos (Cambridge approach), constituído por um conjunto de ações operacionais com seus respectivos indicadores que auxilie numa sequência de atividades que sejam planejadas e organizadas para atender todas as exigências da norma e requisitos regulatórios.

Este artigo foi elaborado no sentido de sistematizar um processo e desenvolver procedimentos operacionais para a aplicação prática da metodologia. Para isto realizaram-se entrevistas com especialistas acadêmicos e não acadêmicos colhendo observações para 
identificar possibilidades de aplicação com base na racionalidade e críticas dos pares.

\section{Referencial teórico}

Esta seção mostra os conceitos de construção para um processo de implantação de um sistema de gestão das operações em organizações da indústria da saúde, orientado pelas normas ISO 13485 e da RDC Nº 59 baseado em conceitos teóricos. Esta posição teórica é utilizada como framework estrutural que guia o desenvolvimento do framework processual.

\subsection{Abordagens por Processos}

A abordagem por processos, também conhecida como Cambridge Approach por ter origem na Universidade de Cambridge, Inglaterra, surgiu quando, em 1990, Ken Platts e Mike Gregory identificaram a necessidade de representar a concepção da estratégia de manufatura. Platts (1994) destaca que o método tem como motivação o desenvolvimento de uma abordagem prescritiva, que operacionaliza um conjunto de conceitos através de um processo estruturado e com instrumentos de coleta de dados, dinâmica e critérios de avaliação. Nessa representação, os professores Ken Platts e Mike Gregory adotaram um conjunto de ferramentas e formulários, denominados Folhas de Tarefa (Worksheets), que auxiliavam no processo de um sistema chamado "Auditoria Estratégica de Manufatura". Estas Folhas de Tarefa eram utilizadas para levantamento das atividades importantes na formulação da estratégia de manufatura.

Ao longo do tempo, o modelo de abordagem por processos de Cambridge evoluiu de forma significativa, desde as primeiras publicações de Platts e Gregory (1990) até as publicações mais recentes de Mills et al (2002). Pinheiro de Lima et al (2009) apresentaram um trabalho que desenvolve um processo de integração da abordagem por processos de Mills et al (2002) com as operações para projetar um sistema de medição de desempenho. A técnica sistematiza os passos para gerar um conjunto de medidas de desempenho com foco nos objetivos estratégicos da empresa e, por conseqüência, uma implementação da estratégia fundamentada em processos e procedimentos. Este framework desenvolve a racionalidade que é a base utilizada para o desenvolvimento da metodologia apresentada neste trabalho.

O framework inicia-se pela definição dos objetivos estratégicos, os quais deverão estar alinhados com as ações que os suportam. A seguir, passa-se à analise das ações e práticas da empresa, confrontando-as com as necessidades exigidas pelo mercado, identificando-se “GAPs”. Tais 'GAPs' conduzem a redefinição de práticas e a elaboração de novas ações. A caracterização das novas ações complementa-se com o desenvolvimento de indicadores 
operacionais ou à revisão / adequação de indicadores existentes, de forma a consolidar o Sistema de Medição de Desempenho (SMD). Folan e Browne (2005) indicam claramente a necessidade de se sistematizar o processo de revisão de indicadores do SMD em geral.

\subsection{Cadeias de valor}

Durante a implantação de um sistema de gestão da qualidade é necessária a criação de procedimentos regulamentares definidos, documentados e implementados de acordo com a norma em questão. Estes procedimentos são divididos pelas diversas atividades da empresa.

A Cadeia de Valor é um sistema de atividades interdependentes conectadas por elos, enquadrando as atividades de valor da empresa em categorias, distribuídas em: atividades primárias e atividades de apoio (ou suporte) (PORTER, 1992).

\subsection{Requisitos das Normas}

A RDC No 59/2000 é similar à Good Manufacturing Practice Regulations (GMP) do órgão americano FDA, que segue todos os requisitos e comprometimento com a qualidade da norma ISO 9001:1994, ou seja, sem enfoque nenhum na estratégia. Já a ISO 13485:2003 é baseada na ISO 9001:2000; no entanto, exclui aspectos como melhoria contínua e satisfação do cliente. A ISO 13485:2003 segue os termos específicos médicos conforme definidos pelo comitê técnico responsável da ISO/TS 210. A Figura 1 ilustra estas diferenças com a ISO 9001:2000.

Figura 1 - Diferenças nos Requisitos RDC No 59/2000 e ISO 13485:2003 com a ISO 9001:2000

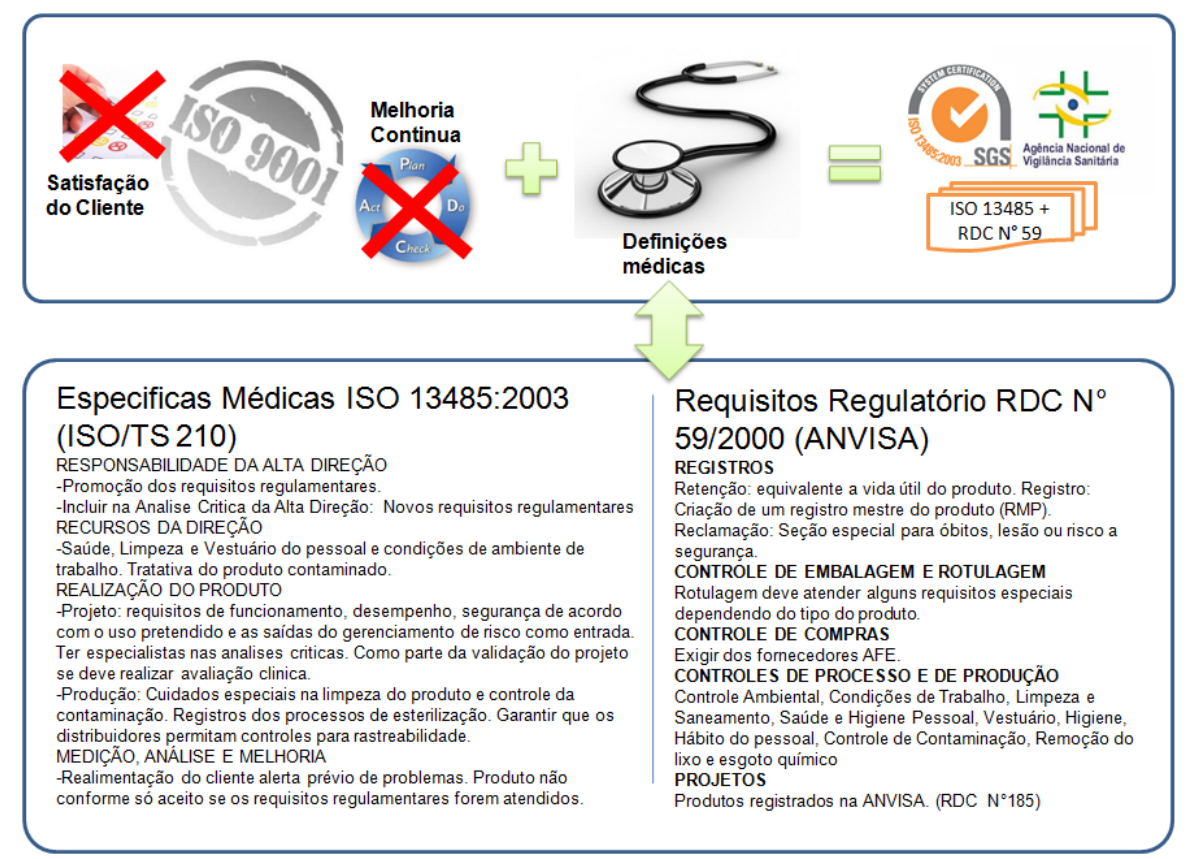

Fonte: Autoria própria (2011) 
Ambas as normas focam mais em aspectos provenientes da segurança do produto, reduzindo riscos de contaminação através de alguns cuidados especiais no controle de processos e produção.

Outro aspecto diferenciado da $\mathrm{RDC}^{\circ}$ 59/2000 é exigir de todos os projetos o registro na ANVISA seguindo a RDC $\mathrm{N}^{\circ} 185$, somente permitindo a comercialização dos produtos após aprovação e liberação no Diário Oficial da União.

No controle de compras, as empresas que fornecem componentes ou serviços relacionados com produtos saneantes domissanitários necessitam Autorização de Funcionamento de Empresas (AFE), permissão para o exercício as atividades sob regime de Vigilância Sanitária.

Outro diferencial importante é a criação de um Registro Mestre de Produto (RMP) para cada projeto registrado na ANVISA. Esse registro deve conter especificações do produto, do processo de produção, de embalagem e rotulagem, protocolo de validação do produto, métodos e procedimentos de instalação, manutenção e assistência técnica.

\subsection{Frameworks de referência}

A metodologia estruturada para implantação de um Sistema de Gestão de Qualidade (SGQ) que atenda os requisitos regulatórios e a norma ISO 13485:2003 foi baseada nos estudos apresentados por Pinheiro de Lima e Gadotti Martins (2011), convergindo aos passos pré-definidos demonstrados na Figura 2. Inúmeros trabalhos foram escritos sobre como implementar SGQs, como os trabalhos de Piskar e Dolinsek (2006), Anholon (2006), Santos (2002), Harr (2001), entre outros. No entanto, poucos estudos têm abordado esta integração com os objetivos estratégicos do negócio e nenhum deles relacionou esta implantação com a RDC N59 e a ISO 13485:2003.

Figura 2 - Metodologia para implantação da RDC N 59/2000 (framework estrutural).

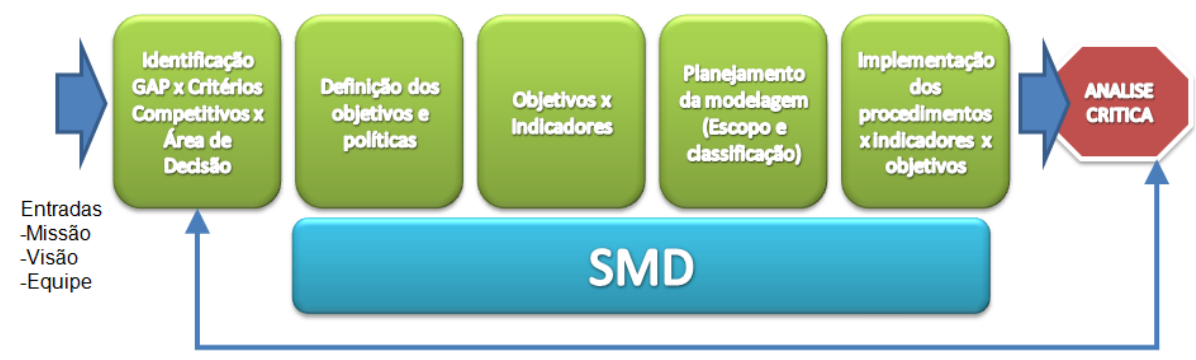

Fonte: Autoria própria (2011)

Para manter uma concordância com a norma, ou seja, um ajuste de vocabulário, neste trabalho as metas de cada área de decisão serão chamadas de objetivos, constituindo o 
objetivo estratégico por área de decisão de política.

Para criar o SGQ integrado à estratégia é necessário inicialmente defini-la ou auditála. O modelo demonstrado no trabalho de Pinheiro de Lima et al (2009) constitui a base para esta integração em termos de definição da estratégia, metas da empresa e indicadores de desempenho. O desempenho é tratado no contexto da estratégia de operações e seu significado é contexto dependente (FOLAN et al, 2007).

Definidos os elementos básicos da estratégia, todas as atividades de controle relacionadas à norma podem ser classificadas, e uma seqüência para modelar e criar procedimentos para todas as atividades regulamentadoras pode ser proposta. Para tanto, o modelo propõe confrontar as metas definidas e as atividades contidas na cadeia de valor de Porter (1992).

O planejamento para a modelagem dos processos (sequiência para descrever os procedimentos operacionais) e implantação segue a literatura, norma e experiência de especialistas em implantação de RDC Nº 59/2000 e ISO 13485:2003.

Atualmente com a globalização, qualquer fato no quadro econômico mundial pode ocasionar alterações no mercado (RUI et al, 2011). Assim, a avaliação da estratégia deve ser reformulada, tal como o SMD (ARTZ et al, 2012, MARINHO e SELIG, 2009). Baseado nestas considerações e nas recomendações do trabalho de Neely et al (1997), o modelo

proposto visa realizar uma análise crítica e contingencial do Sistema de Medição de Desempenho (SMD). Neely (2005) destaca também a necessidade de se manter o SMD constantemente atualizado. Para Bititci et al (2012), o grande desafio atual dos SMD é o desenvolver mecanismos para a revisão de seu projeto, atendendo as demandas de um ambiente em constante mudança.

\section{Metodologia}

Platts (1993) estabelece três critérios principais para avaliação de propostas metodológicas, elaborados através dos questionamentos a seguir, e cujo objetivo é determinar se a metodologia proposta fornece os procedimentos práticos necessários para sua aplicação:

1. Factibilidade: A metodologia proposta é viável e pode ser aplicada na organização?

2. Usabilidade: A metodologia proposta apresenta facilidade de aplicação?

3. Utilidade: A metodologia proposta apresenta utilidade para a organização na solução dos problemas e geração de planos de ação? 
A partir do framework de Pinheiro de Lima e Gadotti Martins (2011) foram conduzidas 5 entrevistas com especialistas acadêmicos e não acadêmicos para refinar o processo e os procedimentos propostos, colhendo observações, possibilidades de aplicação com base na racionalidade e críticas dos pares, seguindo os 3 critérios estabelecidos por Platts (1993).

Para facilitar a compreensão, o processo e os procedimentos serão apresentados e discutidos simultaneamente com a apresentação dos resultados do refinamento.

\subsection{Perfis dos entrevistados}

Foram entrevistados 5 especialistas. As entrevistas tiveram duração entre $1 \mathrm{~h}$ e 1h30min, e foram conduzidas entre Junho e Julho de 2011. Dos 5 entrevistados, 3 são pesquisadores e 2 especialistas na área de qualidade. O Quadro 1 destaca a formação e experiência dos entrevistados.

Quadro 1 - Perfil dos entrevistados

\begin{tabular}{|c|c|c|}
\hline Entrevistado & Formação & Experiência \\
\hline $\begin{array}{c}\text { Pesquisador } \\
1\end{array}$ & $\begin{array}{l}\text { Graduado em Engenharia Industrial } \\
\text { Elétrica pela Universidade Tecnológica } \\
\text { Federal do Paraná (UTFPR-1989), com } \\
\text { mestrado em Engenharia Elétrica } \\
\text { (Automação) pela Universidade Estadual } \\
\text { de Campinas (UNCAMP-1993) e } \\
\text { doutorado em Engenharia (Produção) } \\
\text { pela Escola Politécnica da Universidade } \\
\text { de São Paulo (USP-2003). Doutorado } \\
\text { Sanduíche no Institute for Manufacturing } \\
\text { da Universidade de Cambridge, } \\
\text { Inglaterra (2000-2001). Realizou Pós- } \\
\text { Doutorado no Edward P. Fitts } \\
\text { Department of Industrial and Systems } \\
\text { Engineering da North Carolina State } \\
\text { University (EUA). }\end{array}$ & $\begin{array}{l}\text { Atua principalmente nos seguintes temas: } \\
\text { estratégia de operações (manufatura e } \\
\text { serviços), AMT (advanced manufacturing } \\
\text { technologies), sistemas de medição de } \\
\text { desempenho, operações sustentáveis e Lean } \\
\text { Production. Especialista na aplicação do } \\
\text { process approach treinado pelo prof Ken } \\
\text { Platts no Institute for Manufacturing da } \\
\text { Universidade de Cambridge. }\end{array}$ \\
\hline $\begin{array}{c}\text { Pesquisador } \\
2\end{array}$ & $\begin{array}{l}\text { Graduação em Bacharelado em Estatística } \\
\text { pela Universidade Estadual de Campinas } \\
\text { (1980) e mestrado em Engenharia de } \\
\text { Produção e Sistemas pela Pontifícia } \\
\text { Universidade Católica do Paraná (2005) }\end{array}$ & $\begin{array}{l}\text { Atua principalmente nos seguintes temas: } \\
\text { modelagem de processos de negócios } \\
\text { (BPM), engenharia de software, fábrica de } \\
\text { software, tecnologia P3Tech, processo } \\
\text { contextual, process approach, pesquisa e } \\
\text { desenvolvimento de sistemas } \\
\text { georeferenciados. }\end{array}$ \\
\hline $\begin{array}{c}\text { Pesquisador } \\
3\end{array}$ & $\begin{array}{l}\text { Engenheiro Industrial Eletricista formado } \\
\text { pelo CEFET-PR em 1993. Mestrado em } \\
\text { Informática Aplicada obtido na PUCPR } \\
\text { em } 1999 \text { e Mestrado em Sistemas } \\
\text { Industriais e Engenharia de Produção } \\
\text { obtido pelo Laboratoire d'Analyse et } \\
\text { d`Architecture des Systèmes LAAS, } \\
\text { França, em 2002. Doutorado em } \\
\text { Automação e Sistemas Industriais obtido } \\
\text { pelo LAAS-CNRS, França, obtido em }\end{array}$ & $\begin{array}{l}\text { Ensino, projetos de pesquisa e } \\
\text { desenvolvimento: - Avaliação e projeto de } \\
\text { sistemas de supervisão, controle e avaliação } \\
\text { de desempenho para Sistemas de } \\
\text { Gerenciamento de Produção e da } \\
\text { Manutenção. Avaliação e projeto de } \\
\text { sistemas técnicos de informação no apoio à } \\
\text { Gestão da Produção e da Manutenção. - } \\
\text { Aplicação de técnicas FDD (Fault } \\
\text { Detection and Diagnosis) e Inteligência }\end{array}$ \\
\hline
\end{tabular}




\begin{tabular}{|c|c|c|}
\hline & 2006. & $\begin{array}{l}\text { Artificial aos processos industriais e } \\
\text { organizacionais. }- \text { Sistemas de } \\
\text { Gerenciamento de Processos de Negócio } \\
\text { (Business Process Management - BPMS) e } \\
\text { Workflow. }\end{array}$ \\
\hline $\begin{array}{c}\text { Especialista } \\
1\end{array}$ & 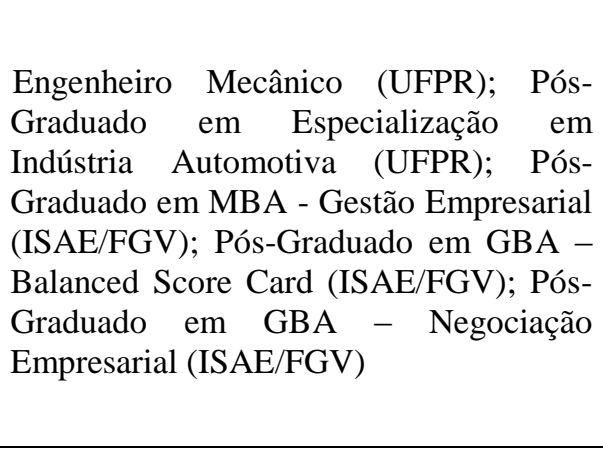 & $\begin{array}{l}\text { Consultoria para implementação de } \\
\text { Programas e Sistemas de Gestão da } \\
\text { Qualidade: ISO 9001, VDA 6.1, EAQF, } \\
\text { ISO/TS-16949, QS 9000, 5 S, PBQP-H e } \\
\text { HACCP; Consultoria para implementação } \\
\text { de Programa de Gestão Ambiental ISO } \\
\text { 14001; Consultoria para implementação da } \\
\text { RDC 59 e da ISO 13485 - Saúde; } \\
\text { Consultoria para implementação da IEC } \\
\text { 17025 - Laboratórios de Ensaio e } \\
\text { Calibração. }\end{array}$ \\
\hline $\begin{array}{c}\text { Especialista } \\
2\end{array}$ & Engenheiro de Qualidade & $\begin{array}{l}\text { Gerente de Qualidade de Indústria de } \\
\text { Ortopedia. Participação de } 2 \text { processos de } \\
\text { implantação das normas ISO } 9001 \text { e } 1 \\
\text { participação na implantação das normas } \\
\text { ISO 9001, ISO 13485, CE e RDC N }{ }^{\circ} 59 \text {. }\end{array}$ \\
\hline
\end{tabular}

Fonte: Autoria própria (2011)

\subsection{Questionário}

Aos entrevistados foi submetido um questionário com as seguintes perguntas básicas, para cada uma das nove etapas propostas pelo framework:

- Factibilidade: A etapa proposta pode ser aplicada?

- Factibilidade: A etapa proposta é de fácil entendimento?

- Usabilidade: A etapa proposta apresenta facilidade na aplicação?

- Utilidade: A etapa proposta atende os objetivos que se propõe?

Para cada uma das perguntas era solicitada, além da crítica dos entrevistados, a apresentação de sugestões.

Além do questionário descrito, houve a apresentação de:

- Definição do problema;

- Objetivo geral e objetivos específicos;

- Representação gráfica do framework;

- Descrição do processo e folhas de tarefa associadas. 


\section{Resultados do refinamento}

As entrevistas não só contribuíram para o aprimoramento do framework processual como também em sua operacionalização de uma forma mais coerente, buscando elaborar um processo que tenha uma aplicação prática nas empresas. Nesta seção serão apresentadas todas as fases e folhas de tarefa do framework refinadas, destacando-se as contribuições dos entrevistados.

\subsection{Principais Contribuições}

Após a análise dos questionários elaborou-se um resumo, apresentado no Quadro 2, com as principais contribuições prestadas pelos entrevistados para o aperfeiçoamento do framework.

Quadro 2 - Principais contribuições prestadas pelos entrevistados

\begin{tabular}{|c|c|}
\hline Etapa do Framework & Contribuição \\
\hline Formular estratégia & $\begin{array}{l}\text { Como se está trabalhando na implantação de um sistema de } \\
\text { gestão para uma empresa na área de saúde, na formulação da } \\
\text { estratégia deve ser analisado os aspectos regulatórios (normas e } \\
\text { regulamentos regional e nacional) para a comercialização do } \\
\text { produto. Estes requisitos são importantes porque podem } \\
\text { interferir tanto no mapeamento dos processos como nos riscos e } \\
\text { requisitos para o desenvolvimento de um produto e/ou modelo } \\
\text { de gestão. Criar uma etapa ou fase entre o Lançamento do } \\
\text { Projeto e Objetivos por Área relacionada ao levantamento dos } \\
\text { requisitos regulatórios. }\end{array}$ \\
\hline Formular estratégia & $\begin{array}{l}\text { Discussão do critério competitivo, desdobrar o que é necessário, } \\
\text { exemplo, o que é qualidade para esta empresa? }\end{array}$ \\
\hline $\begin{array}{l}\text { GAP x Critérios Comp. x } \\
\text { Área de Decisão }\end{array}$ & Alterar áreas de decisão para categoria de decisão. \\
\hline $\begin{array}{l}\text { Definir indicadores } \\
\text { Modelagem dos processos }\end{array}$ & $\begin{array}{l}\text { Observar para que na elaboração dos indicadores não se trabalhe } \\
\text { com fonte de dados muito contextuais. Nestes casos será } \\
\text { necessário analisar a pessoa que irá medir. }\end{array}$ \\
\hline Modelagem dos processos & Para os redatores utilizar quando possível analista de processos. \\
\hline Modelagem dos processos & $\begin{array}{l}\text { Ao invés de utilizar o fluxograma como ferramenta para mapear } \\
\text { o processo deve se trabalhar preferencialmente com o BPMN } \\
\text { que é uma linguagem funcional que atende os requisitos de } \\
\text { processo e setores com um formato gráfico fácil e simples. }\end{array}$ \\
\hline
\end{tabular}

Fonte: Pesquisa de campo (2011)

\subsection{Modelo Processual}

A metodologia processual refinada é apresentada na Figura 3. 
Figura 3 - Modelo processual refinado (framework processual).

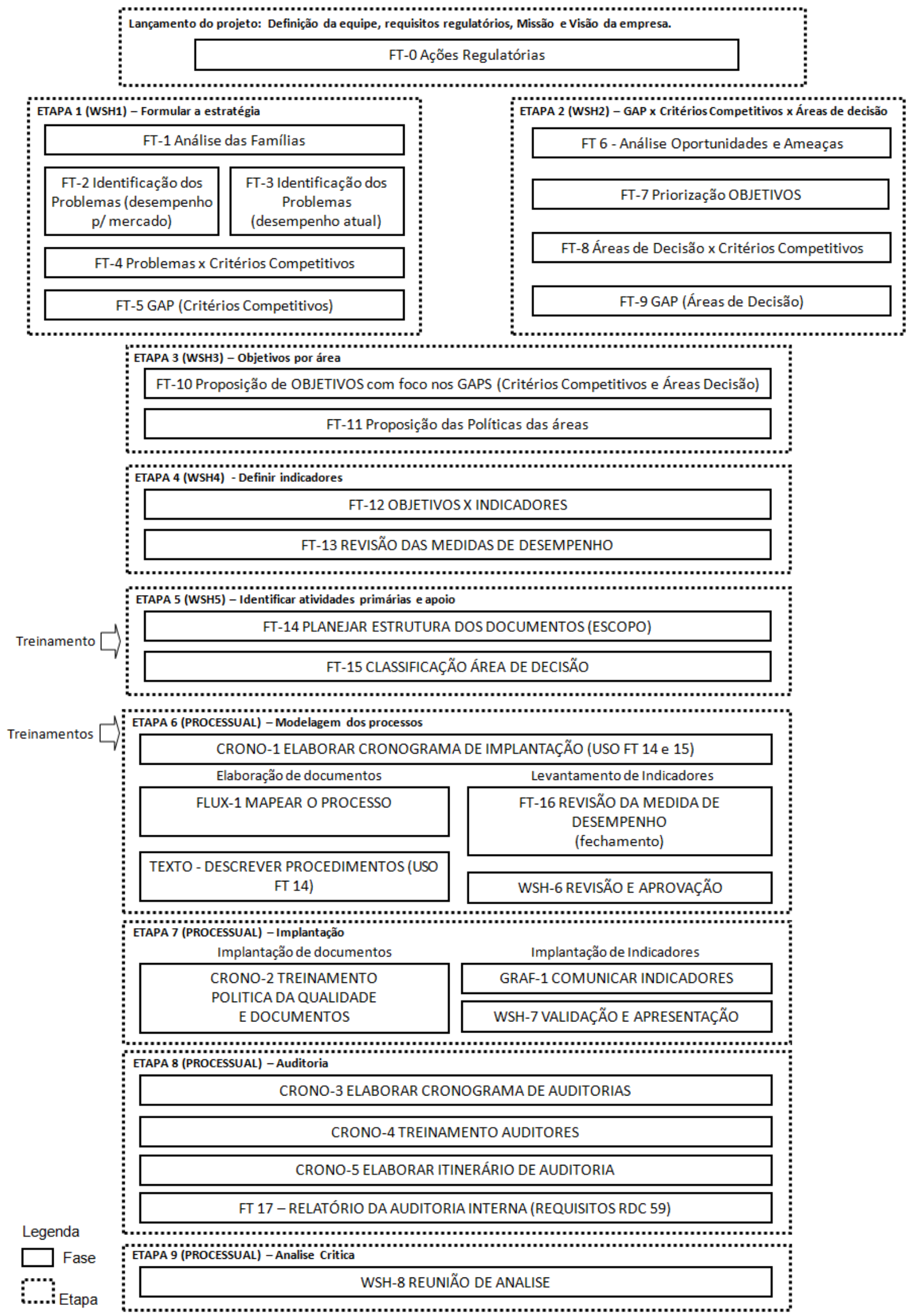

Fonte: Adaptado de Pinheiro de Lima e Gadotti Martins (2011) 
O desenvolvimento do processo no contexto da abordagem por processos de Cambridge apresentado em Platts (1993) e Mills et al (2002), se dá em etapas, além da fase de Lançamento do Projeto, através do preenchimento de folhas de tarefa, doravante denominadas de FTs.

O modelo refinado é dividido em nove etapas, 18 FTs, 5 cronogramas (CRONO), 1 fluxograma de processo (FLUX), 1 modelo de gráfico (GRAF) e seminários (workshops WSH). As primeiras 5 etapas, seguindo o modelo de Mills et al (2002) e Farias et al (2004), são realizadas através de seminários.

O lançamento é a introdução do projeto na empresa, e nada mais é que a venda do projeto para a alta direção que deve "patrocinar" a sua realização (SLACK et al, 2002).

Nesta etapa se deve organizar um grupo de trabalho que será representado pelos seguintes integrantes: o líder (gerente geral do projeto), o representante da direção - RD (gerente do projeto), o grupo de coordenação (gerentes/supervisores), os redatores (responsáveis pela elaboração dos documentos) e os auditores internos.

A participação das equipes distribuídas pelas etapas é sugerida no Quadro 3.

Quadro 3 - Participação sugerida por etapa.

\begin{tabular}{|c|c|c|c|}
\hline Etapas & Descrição & Participação Sugerida & Estratégia \\
\hline 1 & Formular a estratégia & $\begin{array}{l}\text { Diretores, Gerentes, RD e } \\
\text { Supervisores }\end{array}$ & WORKSHOP \\
\hline 2 & $\begin{array}{c}\text { GAP x Critérios Competitivos x } \\
\text { Área de decisão }\end{array}$ & $\begin{array}{l}\text { Diretores, Gerentes, RD e } \\
\text { Supervisores }\end{array}$ & WORKSHOP \\
\hline 3 & Objetivos por área & $\begin{array}{l}\text { Diretores, Gerentes, RD e } \\
\text { Supervisores }\end{array}$ & WORKSHOP \\
\hline 4 & Definir indicadores & Gerentes, RD e Supervisores & WORKSHOP \\
\hline 5 & $\begin{array}{l}\text { Identificar atividades primárias e } \\
\text { secundárias }\end{array}$ & Gerentes, RD e Supervisores & WORKSHOP \\
\hline 6 & Modelagem dos processos & $\begin{array}{c}\text { Gerente, RD, Supervisores e } \\
\text { Redatores }\end{array}$ & $\begin{array}{c}\text { Treinamento, Organização e } \\
\text { Coordenação }\end{array}$ \\
\hline 7 & Implantação & Gerente, RD e Supervisores & Treinamento \\
\hline 8 & Auditoria & RD e Auditores Internos & $\begin{array}{c}\text { Treinamento, Organização e } \\
\text { Coordenação } \\
\end{array}$ \\
\hline 9 & Análise Crítica & $\begin{array}{l}\text { Diretores, Gerentes, RD e } \\
\text { Supervisores }\end{array}$ & Reunião periódica \\
\hline
\end{tabular}

Fonte: Pesquisa de campo (2011)

Ainda nesta etapa devem-se traçar 2 dos 3 determinantes indispensáveis para qualquer empreendimento (MILLS et al, 2002): determinar a missão e visão do negócio com a alta direção. Como características para traçar esta estratégia Mills et al (2002) destacam: um horizonte de longa duração no aspecto tempo, o impacto deve ser significativo, os padrões de decisão devem ser consistentes entre todos os aspectos, o inter-relacionamento efetivo em 
todas as áreas da empresa.

Uma das considerações indicadas pelos especialistas se deu em relação à definição dos requisitos regulatórios, sendo utilizado para tanto, ao final da etapa do lançamento do projeto foi, uma folha de tarefa (FT 0). A escolha pela inserção da FT 0 nesta etapa se deu em virtude de sua possível interferência nas etapas 1 e 2 .

Figura 4 - Folha de tarefa Ações Regulatórias (FT-0).

\begin{tabular}{|l|c|c|}
\hline \multicolumn{2}{|c|}{ FTO - AÇõES REGULATÓRIAS } \\
\hline Requisitos Regulatórios & Competência & Recomendação \\
\hline & & \\
\hline & & \\
\hline
\end{tabular}

Fonte: Autoria Própria (2011)

A primeira etapa 'formular a estratégia' se concretiza no primeiro seminário (WSH1), onde são abordados os objetivos da estratégia para a função operação. Nesta etapa são caracterizados os principais produtos da empresa e os problemas associados a estes, correlacionando-os ao desempenho atual da empresa e às exigências do mercado.

A primeira folha de tarefa procura verificar a posição da empresa em relação ao mercado. Procura-se focar em um número não muito superior a três famílias, se possível (FARIAS et al, 2004).

Figura 5 - Modelo processual proposto.

\begin{tabular}{|l|c|c|c|c|c|}
\hline \multirow{2}{*}{$\begin{array}{c}\text { Familia de } \\
\text { Produtos }\end{array}$} & $\begin{array}{c}\text { Vendas de cada } \\
\text { produto em relação as } \\
\text { vendas totais (\%) }\end{array}$ & $\begin{array}{c}\text { Contribuição do } \\
\text { produto em relação a } \\
\text { contribuição total (\%) }\end{array}$ & $\begin{array}{c}\text { Market share do } \\
\text { produto em relação ao } \\
\text { mercado total (\%) }\end{array}$ & $\begin{array}{c}\text { Crescimento interno } \\
\text { esperado de market } \\
\text { share (fatia do } \\
\text { mercado) }\end{array}$ & $\begin{array}{c}\text { Crescimento esperado } \\
\text { do produto no } \\
\text { tamanho do mercado } \\
\text { total }\end{array}$ \\
\hline Produto 1 & & & & & \\
\hline Produto 2 & & & & & \\
\hline
\end{tabular}

Fonte: Autoria Própria (2011)

Após o posicionamento da empresa, passa-se à folha de tarefa 2, onde serão identificados possíveis problemas em relação ao desempenho esperado pelo mercado, relacionando-os aos critérios competitivos, tal como é percebido pela empresa em atenção às exigências de mercado. Usando a escala de LIKERT a percepção para esta folha de tarefa fica: desconhecida por todos [-2], desconhecida parcialmente [-1], neutralidade [0], conhecida por todos [+1], muito bem conhecida por todos [+2].

Por fim, a folha de tarefa 3 identifica problemas em relação ao desempenho atual da 
empresa, etapa observada após a identificação dos critérios competitivos e do posicionamento da empresa em relação ao mercado.

Como visto, as FT 2 e FT 3 são similares, diferenciando-se apenas na modalidade da exigência/desempenho a ser identificado, sendo a primeira em relação à expectativa exigida pelo mercado, e a segunda quanto ao desempenho atual da empresa.

Os critérios competitivos que são requeridos pelo mercado são relacionados por Slack et al(2002) como custo, qualidade, tempo, flexibilidade e inovação, atentando-se aos efeitos internos e externos. Nesta fase, deve ser definido claramente na folha de tarefa todos os 5 critérios competitivos, outra contribuição especificada pelos especialistas.

Figura 6 - FT 2 (Identificando problemas - exigências do mercado)

\begin{tabular}{|c|c|c|c|c|c|c|}
\hline \multicolumn{7}{|c|}{$\begin{array}{c}\text { FT2 - IDENTIFICAÇÃO DOS PROBLEMAS NA ATUAL FAMILIA DE PRODUTOS } \\
\text { (desempenho exigido pelo mercado) }\end{array}$} \\
\hline \multicolumn{7}{|c|}{$\begin{array}{l}\text { "Qualidade: ao 'fazer as coisas certas', a produção procura influenciar a qualidade dos bens e serviços da empresa. } \\
\text { "Rapidez / Velocidade: ao 'fazer as coisas mais rapidamente', a produção procura influenciar a velocidade com que os bens e serviços são fornecidos. } \\
\text { "Custos: para empresas que concorrem diretamente em preços, o custo será seu principal objetivo. Quanto menor o custo de seus bens produzidos, ou de } \\
\text { seus serviços prestados, menor poderá ser o preço fornecido aos seus clientes / consumidores. } \\
\text { "Fonfiabilidade: ao 'fazer as coisas a tempo', a produção procura influenciar a confiabilidade de entrega de bens e serviços. } \\
\text { "Flexibilidade: a capacidade de 'mudar o que e como se faz', a produção procura influenciar a flexibilidade, com a qual a empresa produz bens e serviços. }\end{array}$} \\
\hline \multirow{2}{*}{$\begin{array}{l}\text { Família de } \\
\text { Produtos }\end{array}$} & \multirow{2}{*}{ Preço } & \multirow{2}{*}{ Qualidade } & Tempo & \multicolumn{2}{|c|}{ Flexibilidade } & \multirow{2}{*}{ Confiabilidade } \\
\hline & & & Lead Time & de produto & de Volume & \\
\hline \multicolumn{7}{|l|}{ Produto 1} \\
\hline Produto 2 & & & & & & \\
\hline
\end{tabular}

Fonte: Autoria Própria (2011)

Confrontando os resultados nas FT2 e a FT3 obtém-se como resultado os GAP, usando o 'método de perfis', conforme apresentado na Figura 7.

Figura 7 - FT 4 (Identificando problemas - problemas x critérios competitivos)

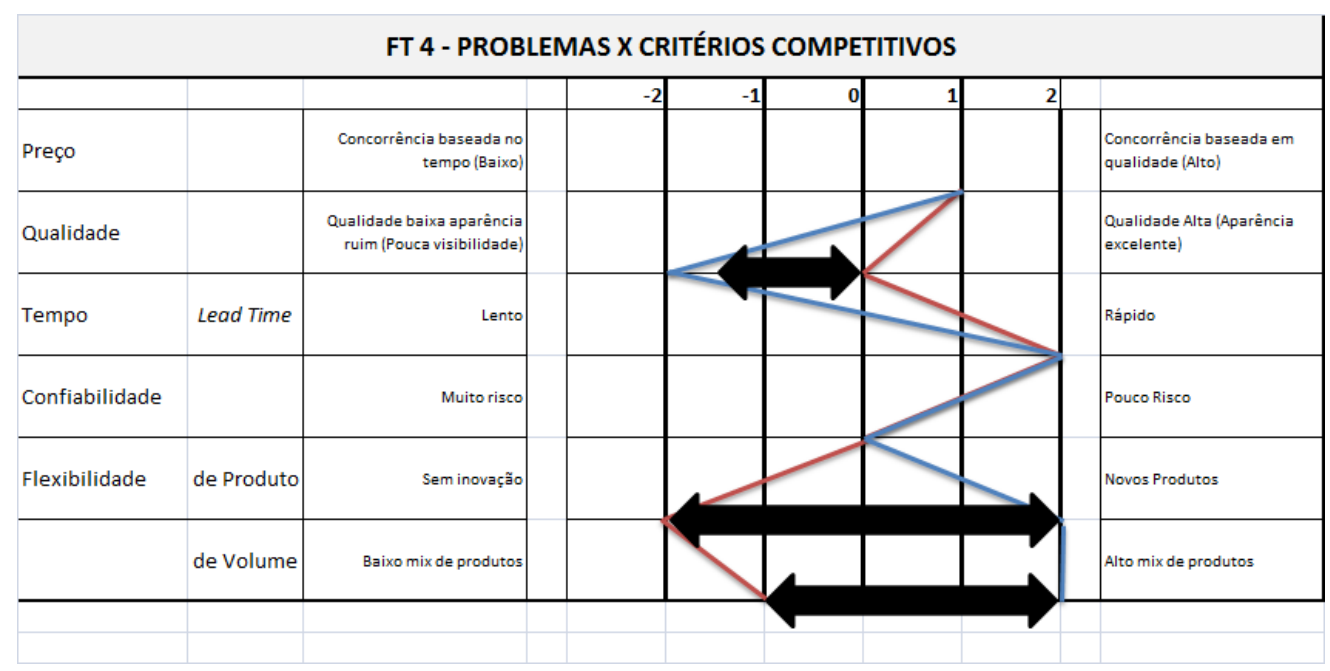

Fonte: Autoria Própria (2011) 
O WSH1 é finalizado com a descrição dos GAP associados aos critérios competitivos que devem ser estabelecidos (FT 5).

O WSH2 é aplicado às FT6 à FT9, etapa em que são identificadas as atuais ações e práticas da empresa e seus impactos nos resultados.

A FT 6 identifica as tendências de mercado, descrevendo as oportunidades e ameaças externas.

Figura 8 - FT 6 (Tendências do mercado)

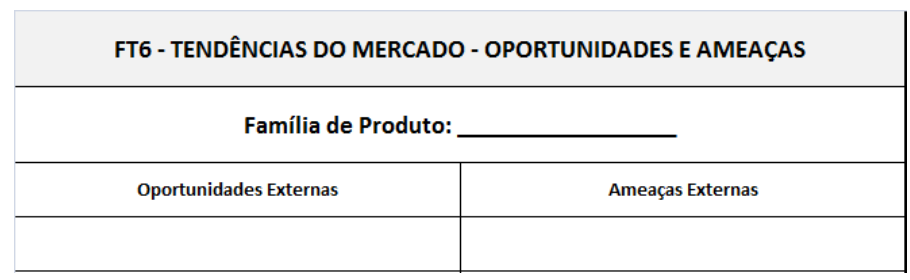

Fonte: Autoria Própria (2011)

Em seguida, utilizando os dados apresentados na FT 6, estabelece-se a priorização dos objetivos, possibilitando o preenchimento da FT 7 para cada família de produto, iniciando pelas colunas (1) e (2), concluindo com a coluna (3).

Figura 9 - FT 7 (Priorização dos objetivos do negócio.)

\begin{tabular}{|l|c|c|c|}
\hline \multicolumn{5}{|c|}{ FT7 - PRIORIZAÇÃo DOS OBJETIVOS DOS NEGÓCIOS } \\
\hline $\begin{array}{l}\text { Família de } \\
\text { Produto }\end{array}$ & $\begin{array}{c}\text { Necessidade dos cliente } \\
(1)\end{array}$ & Objetivos / Metas (3) & $\begin{array}{c}\text { Necessidades dos } \\
\text { Stakeholders (2) }\end{array}$ \\
\hline Produto 1 & & & \\
\hline
\end{tabular}

Fonte: Autoria Própria (2011)

O próximo passo é relacionar as ações e as práticas atuais da empresa na FT 8 , cruzando as categorias de decisão e os critérios competitivos (CORREA, 1994). Nesta fase foi alterada a palavra área de decisão para categoria de decisão, conforme contribuição dos especialistas.

Figura 10 - FT 8 (Categorias de decisão x Critérios Competitivos)

\begin{tabular}{|c|c|c|c|c|c|c|}
\hline \multicolumn{7}{|c|}{ FT8 - CATEGORIA DE DECISÃo X CRITÉRIOS COMPETITIVOS } \\
\hline $\begin{array}{c}\text { Categoria de } \\
\text { decisão }\end{array}$ & Prática Atual & Custo & Qualidade & Tempo & Flexibilidade & $\begin{array}{c}\text { Confiabilida } \\
\text { de }\end{array}$ \\
\hline Instalações & & & & & & \\
\hline
\end{tabular}

Fonte: Autoria Própria (2011) 
Novamente, a FT 8 será preenchida utilizando-se da escala LIKERT (-2 à +2) de acordo com a sensibilidade do colaborador. As categorias de decisão são classificadas conforme apresentado no trabalho de Pinheiro de Lima et al (2008).

A FT9 encerra a etapa, onde serão registrados os GAPs extraídos da FT8 em relação aos objetivos competitivos (FT7), consolidando aqueles entre o desempenho atual e o exigido pelo mercado nas dimensões relacionadas às áreas de decisão.

Com os GAPs elaborados com os WSH1 e WSH2, têm-se elementos para propor objetivos para o negócio. Conforme citado pela RDC No 59/2000 é necessário que cada fabricante estabeleça sua política e seus objetivos de comprometimento com a qualidade, para então na etapa 3 - objetivos por área WSH3, FT10 e FT11 - serem definidos objetivos e políticas dos negócios relacionados com cada área de decisão da empresa.

Figura 11 - FT 11 (Políticas do negócio)

\begin{tabular}{|c|c|c|}
\multicolumn{3}{|c|}{ FT11 - POLITICAS DO NEGÓCIO } \\
\hline Área de decisão & objetivos & Política da área \\
\hline \multirow{2}{*}{ Qualidade } & $01-\ldots$. & \\
& $03-\ldots$. & \\
$04-\ldots$. & \\
\hline Produção & $02-\ldots$. & \\
\hline
\end{tabular}

Fonte: Autoria Própria (2011)

A etapa 4 (Definir indicadores), também é realizada na forma de workshop (WSH4) onde são aplicadas as FT12 e FT13. Já dispondo dos objetivos/metas é necessário que sejam identificados os indicadores operacionais associados, visando a medição de desempenho e a monitoração da eficácia no alcance dos objetivos e metas estabelecidos.

A partir disto o modelo de Neely et al (1997), chamado "a structured approach", tem como objetivo responder a seguinte questão: O que uma medida de desempenho bem concebida deve constituir? Sob esta perspectiva a etapa 4 propõe, através da FT12, levantar os indicadores provenientes dos objetivos gerados, e pela FT 13, facilitar a concepção de medidas de desempenho e encorajar os projetistas de tais medidas a considerarem as implicações do comportamento das medidas em configurações específicas.

Por serem constantes as problemáticas envolvendo fontes de dados e pontos de coleta, o que geralmente está associado à falta de credibilidade e/ou falta transparência destas informações, a FT13 deverá ser preenchida integralmente à exceção do campo "fonte de dados". 
Figura 12 - FT 12 (Políticas do negócio)

\begin{tabular}{|l|c|}
\hline \multicolumn{2}{|c|}{ FT12 - OBJETIVOS X INDICADORES } \\
\hline obejtivos (FT 10) & Inclusão / alteração de INDICADORES \\
\hline & \\
\hline
\end{tabular}

Fonte: Autoria Própria (2011)

Figura 13 - FT 13 (Revisão da medida de desempenho).

\begin{tabular}{l|l|}
\multicolumn{2}{|c|}{ FT 13 - REVISÃO DA MEDIDA DE DESEMPENHO } \\
\hline \multicolumn{1}{|c|}{ OBJETIVO DO NEGÓCIO: } \\
\hline Titulo: & \multicolumn{1}{|c|}{ Indicador: } \\
\hline Proposta: & \\
\hline Monitorar o: & \\
\hline Meta: & \\
\hline Formula: & \\
\hline Frequência: & \\
\hline Quem Mede: & \\
\hline $\begin{array}{l}\text { Fontes de Dados: } \\
\text { Quem atua sobre a } \\
\text { medida: }\end{array}$ & \\
\hline O que fazer com os \\
dados?
\end{tabular}

Fonte: Adaptado de Neely (1997)

O objetivo da etapa 5 é classificar os procedimentos a serem elaborados para identificar uma seqüência para a etapa de modelagem.

Na primeira fase desta etapa devem ser definidos o escopo, normas e requisitos legais aplicáveis, sendo necessário treinamento sobre requisitos da norma RDC N59. Através da FT 14, o planejamento da estrutura de documentos deve relacionar a área de decisão, setor responsável, política da área (para os casos em que não for preciso escolher uma relacionada com a área, ao exemplo da política de qualidade, necessária em toda a empresa), objetivo (se existir) e documentos a serem elaborados e indicadores (quando existir). A intenção é identificar tudo que está relacionado com os documentos para o desenvolvimento da etapa modelagem. 
Figura 14 - FT 14 (Planejar Estrutura de Documentos)

\begin{tabular}{|c|c|c|c|c|c|}
\hline \multicolumn{6}{|c|}{ FT 14 - PLANEJAR ESTRUTURA DE DOCUMENTOS } \\
\hline \multicolumn{6}{|c|}{ Há exclusão para o negócio: ___ (itens da norma) } \\
\hline Área de Decisão & Setor Responsável & Política & Objetivos & Documentos & Indicadores \\
\hline $\begin{array}{c}\text { Sistema de } \\
\text { melhoria continua }\end{array}$ & \multirow[t]{2}{*}{ Qualidade } & Política de Qualidade & $\begin{array}{l}01-\ldots \\
02-\ldots\end{array}$ & $\begin{array}{l}\text { * Procedimento: Procedimento 1, } \\
\text { Procedimento } 2 . \\
\text { * Registros: Registro 1 } \\
\text { * Formulários: Controle 1, Formulário } 2\end{array}$ & $\begin{array}{l}\text { Indicador } 1 \\
\text { Indicador } 2\end{array}$ \\
\hline $\begin{array}{l}\text { Politicas de } \\
\text { Qualidade }\end{array}$ & & Política de Qualidade & $\begin{array}{l}01-\ldots \\
02-\ldots\end{array}$ & $\begin{array}{l}\text { * Procedimento: Procedimento } 1 \text {, } \\
\text { Procedimento } 2 . \\
\text { * Registros: Registro } 1\end{array}$ & $\begin{array}{l}\text { Indicador } 1 \\
\text { Indicador } 2\end{array}$ \\
\hline
\end{tabular}

Fonte: Autoria Própria (2011)

Depois de relacionados os documentos, indicadores e objetivos com a área de decisão, é preciso classificar a área de decisão, a qual servirá posteriormente de referência para a etapa de modelagem. Para tanto se utilizará na FT 15 a idéia de cadeia de valor de Porter (1992).

Figura 15 - FT 15 (Classificação Áreas de Decisão). Fonte: Adaptado de Porter (1992).

\begin{tabular}{|c|c|}
\hline \multicolumn{2}{|c|}{ FT 15 - CLASSIFICAÇÃo ÁREAS DE DECISÃo } \\
\hline $\begin{array}{c}\text { ATINIDADES } \\
\text { PRIMÁRIAS }\end{array}$ & \\
\hline $\begin{array}{c}\text { ATIVIDADES } \\
\text { SECUNDÁRIAS }\end{array}$ & \\
\hline
\end{tabular}

Fonte: Autoria Própria (2011)

A etapa 6 não acontece mais no formato de workshop, visto que além do processo de modelagem dos documentos demandar mais tempo, suas fases são realizadas por um único colaborador ou um pequeno grupo de colaboradores. Igualmente, esta etapa requer alguns dados de entrada: treinamento de elaboração de documentos, treinamento de mapeamento de processos e treinamento de requisitos da RDC N 59 e ISO 13485:2003.

A primeira fase desta etapa é elaborar o cronograma de modelagem, implantação e auditoria interna (CRONO1), que tem como base a FT14 (escopo) e a FT15, supramencionadas.

Ainda nesta etapa, o fluxo é subdividido em dois caminhos: elaboração de documentos e a conclusão do levantamento de indicadores.

$\mathrm{Na}$ elaboração de documentos da área faz-se necessário inicialmente mapear o processo. Para tanto, deve ser utilizado preferencialmente o BPMN (Business Process Modeling Notation), conforme indicado nas contribuições dos especialistas porque, ao contrário de um fluxograma simples, o BPMN atende tanto requisitos de processo como de setores. Após o mapeamento inicia-se a fase de descrever os procedimentos, registros e 
formulários dos processos relacionados na FT 14. Esta fase, a ser realizada pelo redator, será encaminhada ao Supervisor/Gerente da área de decisão para revisão e aprovação.

Paralelo à fase de elaboração dos documentos, deverá o Supervisor/Gerente da área de decisão preencher a FT 16, que nada mais é que a conclusão da FT 13 através do preenchimento do campo "fonte de dados". Referidos dados são de suma importância porque a partir deles será criado o registro e/ou implantação de sistemas para medir o objetivo. Ao final da FT 16, submeter-se-á à aprovação da Diretoria através de um seminário a fim de avaliar a compatibilidade da fonte de dados sugerida com o objetivo.

A etapa 7 consiste na implantação de todos os dados, indicadores e documentos criados. Novamente 2 fluxos paralelos de ações são realizados: implantação de documentos e implantação de indicadores.

O fluxo de implantação de documentos inicia com a fase de treinamento da política da qualidade, manual da qualidade e procedimentos, os quais são gerenciados através de um plano de treinamento (CRONO2). A partir disto os documentos devem ser seguidos por todos colaboradores.

O fluxo de implantação de indicadores é iniciado a partir das reflexões: qual a melhor forma de entregar a mensagem? Qual é o público alvo e o que eles querem? Quais são os melhores canais para entregar a mensagem? É, portanto, prudente, uma vez que algumas idéias foram identificadas, sejam apresentadas em um canal de distribuição adequado para o público que se dirigem. Sendo assim, o fluxo tem inicio na fase comunicação na qual se deve criar um modelo gráfico dos indicadores (GRAF1).

É importante ressaltar que a verificação garante a credibilidade dos dados. Por vezes, os dados extraídos podem estar incorretos. Em assim ocorrendo, é na próxima fase a ser realizada que se fará a análise dos dados pela supervisão/gerência a fim de garantir esta credibilidade. Robinson e Stern (1998) sugerem que o alinhamento estratégico é alcançado quando os interesses e as ações de todos os funcionários da empresa estão focados nos objetivos principais da empresa. Sendo assim, a última fase do modelo gráfico dos indicadores pode ser apresentada (WSH6) pelos gestores/gerência periodicamente à direção a fim de garantir o alinhamento da equipe, garantindo desta forma que o SMD tenha um ciclo de melhoria e que realmente seja eficaz. Farris et al(2011) reforça a necessidade de uma abordagem estruturada para gestão da melhoria, enquanto que Fried (2010) destaca o papel da aprendizagem cumulativa neste processo.

A etapa 8 é a de auditoria, um exame estabelecido, sistemático e independente de todo sistema de qualidade, executado em intervalos regulares e com frequiência suficiente para 
assegurar que, tanto as atividades do sistema de qualidade quanto seus resultados, sejam implementados eficientemente e de forma adequada a alcançar os objetivos do sistema de qualidade inicialmente definido.

A primeira fase da etapa é estabelecer um cronograma de auditorias anual (CRONO3), para gerenciar estas avaliações do sistema. Esta fase deve ser elaborada pelo RD juntamente com a Direção. Na seqüência, deve-se iniciar o treinamento do grupo de auditores internos com os requisitos das normas, e para gerenciar esta fase é utilizado um cronograma de treinamento representado no framework processual como CRONO4. Na terceira fase o RD deve elaborar o itinerário da auditoria e divulgar a toda a empresa. Este cronograma (CRONO5) deve iniciar por uma reunião de abertura com supervisores/gerentes/direção para apresentação do itinerário de auditoria e ser encerrado com uma reunião de fechamento apresentando a FT17.

A fase auditoria consiste em preencher a FT 17 que relaciona os requisitos da norma com as não-conformidades encontradas durante o processo de auditoria.

Ações corretivas devem ser realizadas após o preenchimento da FT 17, conforme foi descrito no procedimento criado na etapa modelagem dos processos.

Passando para a etapa 9, de análise crítica, deverá ser definida uma periodicidade de avaliação da estratégia e/ou do SMD, através de uma reunião da direção e gestão (WSH8) que deve 1) verificar os indicadores atuais; 2) comparar as metas e objetivos estratégicos com o mercado atual, ou seja, girando o ciclo novamente iniciando pela etapa 1. Este último deve ser avaliado numa periodicidade maior, ou se algum fator do mercado demonstrar alterações do cenário que careçam reavaliar a estratégia.

Devem ser utilizados como dados de entrada para estas reuniões as ações preventivas, corretivas e os indicadores de desempenho.

\section{Recomendações para aplicação em campo}

As várias etapas e fases do método tornam sua aplicação muito rigorosa e desgastante. Nas contribuições dos entrevistados/especialistas várias recomendações foram sugeridas para orientar esta aplicação e seus efeitos.

1) Etapa Lançamento do Projeto: $\mathrm{Na}$ escolha dos redatores devem ser utilizados preferencialmente Analistas de Processo, porque estes são profissionais qualificados para o mapeamento e/ou desenho dos processos, procurando assim integrar os objetivos organizacionais com os objetivos dos processos da cadeia de valor da empresa. 
2) Etapa Formular Estratégia: A compreensão dos critérios competitivos é importante para fazer convergir as respostas dos entrevistados ao objetivo real do critério. Por exemplo, no critério qualidade, o que é qualidade para nossa empresa?

3) Definir indicadores e Modelagem dos processos: O cuidado para a escolha da fonte de dados deve ser o menos subjetivo possível. Para o caso de ser a fonte de dados contextual, será necessário analisar o individuo que irá medi-la, uma vez que tudo em sua volta poderá afetar o resultado do indicador. Exemplos disso é o humor do individuo, problemas familiares e etc.

4) Execução do Workshop: Durante a implantação devem-se unificar algumas etapas de seminários evitando várias reuniões que podem reduzir a motivação e engajamento.

\section{Conclusão e recomendações para a condução do processo}

O refinamento contribuiu para melhorar aspectos como a factibilidade e utilidade do processo, atendendo de forma mais assertiva aos objetivos que se propõe, facilitando o entendimento e criando melhores condições para o seu teste e aplicação.

A inclusão de uma fase de "Ações Regulatórias" dentro da etapa do lançamento do projeto é um bom exemplo da melhora na utilidade, pois se o objetivo da metodologia é transpor o objetivo estratégico da empresa para todo o sistema de gestão da qualidade, na indústria da saúde o objetivo estratégico muitas vezes está integrado de maneira compulsória a legislação regulatória da região ou país.

De igual forma, para melhoria da aplicação, várias contribuições foram indicadas tais como a preocupação da fonte de dados na elaboração dos indicadores serem muito contextuais, utilização de analistas de processo para serem redatores e dicas de ferramentas mais eficazes para o mapeamento do processo.

O entendimento, como citado, também foi aperfeiçoado com a mudança de "áreas de decisão" para "categorias de decisão".

Assim as entrevistas com os especialistas realmente contribuíram sobremaneira neste processo. Logicamente, não se pode ainda concluir que a metodologia proposta fornece os procedimentos práticos necessários para sua aplicação, mas desde já se observou que a metodologia se aperfeiçoou de modo a possibilitar sua aplicação prática. 


\begin{abstract}
The technical regulations are established by governments through a specific agent in order to ensure the health and safety of users of products for medical use. The adaptation of equipment manufacturers and their standards and technical regulations encourages qualitative improvements in products and manufacturing processes. The objective of this research is to systematize a process already proposed for implementing a system for managing operations in the health industry organizations guided by ISO 13485 and RDC $\mathrm{N}^{\circ}$. 59 developing operational procedures for the practical application. This implementation meets the demands of regulatory agencies and the system integrates the company's strategic objectives. The method is developed using the process approach (Cambridge approach), consisting of a set of operational actions with their respective indicators, assist in a sequence of activities that are planned and organized to meet all requirements of the standard and regulatory requirements. For this were conducted interviews with academic experts and non-academic, registering observations, application possibilities based on rationality and critics. Logically, it can not be yet concluded that the proposed methodology provides the procedures necessary for their practical application, but now it was observed that the methodology has been perfected so as to allow for its application, for further development, in a case study.
\end{abstract}

Key-words: RDC No. 59; Process approach; ISO 13485; Regulatory Requirements; Operations Management

\title{
Referências
}

ANDREWS, K.R. The Concept of Corporate Strategy. Irwin, Homewood, IL, 1971.

ALBUQUERQUE, E.; CASSIOLATO, J. As Especificidades do Sistema de Inovação do Setor Saúde: Uma resenha da Literatura como Introdução a uma Discussão sobre o Caso Brasileiro. Estudos FeSBE I. São Paulo Universidade de São Paulo, 2000.

ANHOLON, R. Método de Implantação de Práticas de Gestão da Qualidade para Microempresas. Programa de Pós-Graduação em Engenharia de Produção. Porto Alegre, 2002.

ARTZ, M.; HOMBURG, C.; RAJAB T. Performance-measurement system design and functional strategic decision influence: the role of performance-measure properties. Accounting, Organizations and Society, v. 37 n. 7, p. 445-460, 2012.

cross ref

BASLER, R.; RUELNAD, R. P. N. The arrival of ISO 13485:20031. Medical Product Outsourcing, January/February 2004.

BITITCI, U.; GARENGO, P.; DÖRFLER, V.; NUDURUPATI, S. Performance measurement: challenges for tomorrow. International Journal of Management Reviews, v. 14, n. 3, p. 305-327, 2012.

cross ref

COELHO, P. M. B. Boas Práticas de Fabricação: Estudo de Caso - Tecnofile. Escola Pública de Saúde do Ceará. Fortaleza, 2008.

CRUZ, A. G.; CENCI, S. A.; MAIA, M. C. A. Quality assurance requirements in produce processing. Trends in Food Science \& Technology, v. 17. n. 8, p.406-411, 2006.

\section{cross ref}

FARIAS, A.R., GOUVEA DA COSTA, S.E., PINHEIRO DE LIMA, E. Metodologia para operacionalizar a estratégia de operações em empresas prestadoras de serviço aplicando a abordagem por processos de Cambridge. In. ENCONTRO NACIONAL DE ENGENHARIA DE PRODUÇÃO, 24, 2004, Florianópolis. Anais... 
Florianópolis, ABEPRO, 2004.

FARRIS, J. A.; VAN AKEN, E. M.; LETENS, G.; CHEARKSUL, P.; COLEMAN, G. Improving the performance review process: A structured approach and case application. International Journal of Operations \& Production Management, v. 31, n. 4, p. 376-404, 2011.

cross ref

FERNANDES, D. R. Uma contribuição sobre a construção de indicadores e sua importância para a gestão empresarial. Revista FAE, Curitiba, v.7, n.1, p.1-18, jan./jun. 2004.

FERREIRA, A. H. Estratégia competitiva: uma análise sistêmica do modelo de Porter e suas implicações nas estratégias de diferenciação do setor laticínios. In. ENCONTRO NACIONAL DE ENGENHARIA DE PRODUÇÃO, 19, 1999, Rio de Janeiro. Anais... Rio de Janeiro: ABEPRO, 1999.

FOLAN, P.; BROWNE, J. A review of performance measurement: towards performance management. Computers in Industry, v. 56, n. 7, p. 663-680, 2005.

\section{cross ref}

FOLAN, P.; BROWNE, J.; JAGDEV, H. Performance: its meaning and content for today's business research. Computers in Industry, v. 58, n. 7, p. 605-620, 2007.

\section{cross ref}

FRANCO-SANTOS, M.; KENNERLEY, M.; MICHELI, P.; MARTINEZ, V.; MASON, S.; MARR, B.; GRAY, D.; NEELY, A. D. Towards a definition of a business performance measurement system. International Journal of Operations \& Production Management, v. 27, n. 8, p. 784-801, 2007.

\section{cross ref}

FRIED, A. Performance measurement systems and their relation to strategic learning: a case study in a softwaredeveloping organization. Critical Perspectives on Accounting, v. 21, n. 2, p. 118-133, 2010.

\section{cross ref}

GADELHA, C. A. G.; QUENTAL, C.; FIALHO, B. C. Saúde e inovação: uma abordagem sistêmica das indústrias da saúde. Cad. Saúde Pública, v. 19, n. 1, p. 47-59, 2003.

\section{cross ref}

GARCIA, L. M.; COSTA L. E. Resumo sob RDC n59/2000 - uma Óptica Prática e Visão Estratégica na Gestão Empresarial. Revista Fármacos \& Medicamentos, v. 55, 2008.

GIMBERT, X.; BISBE, J.; MENDOZA, X. The role of performance measurement systems in strategy formulation processes. Long Range Planning, v. 43, n. 4, p. 477-497, 2010.

\section{cross ref}

HARR, R. TQM in dental practice. Internacional Journal of Health Care Quality Assurance, v. 14, n.2, p. 69-81, 2001

\section{cross ref}

HENRY, J. F. Management control systems and strategy: a resource-based perspective. Accounting, Organizations and Society, v. 31, n. 6, p. 529-558, 2006.

\section{cross ref}

IPPOLITI, R.; FALAVIGNA, G. Efficiency of the medical care industry: Evidence from the Italian regional system. European Journal of Operational Research, v. 217, n. 3, p. 643-652, 2012. 
MARINHO, S. V.; SELIG, P. M. Análise comparativa do Balanced Scorecard com alguns dos principais sistemas de medição de desempenho. Revista Gestão Industrial, v. 5, n. 3, p. 177-197, 2009.

cross ref

MILLS, J.; PLATTS, K.; NEELY, A. D. Creating a Winning Business Formula. Inglaterra. Cambridge University, 2002.

MINISTÉRIO DA SAÚDE. Complexo Industrial. Disponível em: <http://portal.saude.gov.br/portal/saude>. Acesso em: Julho 2009.

MINISTÉRIO DA SAÚDE. ANVISA. RESOLUÇÃO - RDC Nº. 32, DE 29 DE MAIO DE 2007.

MINISTÉRIO DA SAÚDE. ANVISA. RESOLUÇÃO - RDC Nº. 59, DE 27 DE JUNHO DE 2000.

NEELY, A. D. The evolution of performance measurement research: developments in the last decade and a research agenda for the next. International Journal of Operations and Production Management, v. 25, n.12, p. 1264-1277, 2005.

cross ret

NEELY, A. D.; JARRAR, Y. Extracting value from data - the performance planning value chain. Business Process Management Journal, n.10, n.5, p. 506-509, 2004.

cross ref

NEELY, A. D.; GREGORY, M.; PLATTS, K. W. Performance measurement system design: A literature review and research agenda. International Journal of Production Economics, v.15, n. 4, p. 80-116, 1995.

NEELY, A. D.; RICHARDS, H.; MILLS, J. F.; PLATTS, K. W.; BOURNE, M. C. S. Designing performance measures: a structured approach. International Journal of Operations \& Production Management, v. 17, n. 11, p. 1131-1152, 1997.

cross ref

NUDURUPATI, S. S.; BITITCI, U. S.; KUMAR, V.; CHAN, F. T. S. State of the art literature review on performance measurement. Computers and Industrial Engineering, v. 60, n. 2, p. 279-290, 2011.

cross ref

PINHEIRO DE LIMA, E., GADOTTI MARTINS, E.. Proposição de uma metodologia para implantação de RDC 59. In: ENCONTRO NACIONAL DE ENGENHARIA DE PRODUÇÃO, 31, 2011, Belo Horizonte. Anais... Belo Horizonte: ABEPRO, 2011.

PINHEIRO DE LIMA, E.; GOUVEA DA COSTA, S. E.; REIS DE FARIA, A. Taking operations strategy into practice: Developing a process for defining priorities and performance measures. International Journal Production Economics, v. 122, n. 1, 2009.

cross ref

PINHEIRO DE LIMA, E.; GOUVEA DA COSTA ANGELIS, J. J. Strategic Management System Design Process. Brazilian Journal of Oerations \& Production Management, v. 5, n. 1, 2008.

PISKAR, F.; DOLINSEK, S. Implementation of the ISO 9001 from QMS to business model. Industrial Management \& Data Systems, v. 106, n. 9, p. 1333-1343, 2006.

cross ref

PLATTS, K. W.; GREGORY, M. Manufacturing audit in the process of strategy formulation. International Journal of Operations \& Production Management, v.10, n. 9, p. 5-26, 1990. 
PLATTS, K. W. A process approach to researching manufacturing strategy. International Journal of Operations \& Production Management, n.13, n.8, p. 4-17, 1993.

cross ref

PLATTS, K. W. Characteristics of methodologies for manufacturing strategy formulation. Computer Integrated Manufacturing Systems, v.7, n. 2, p. 93-99, 1994.

cross ref

PLATTS, K.W.; MILLS, J.F.; NEELY, A.D.; GREGORY, M.J.; RICHARDS, A.H. Evaluating manufacturing strategy formulation processes. International Journal of Production Economics, V.46-47 N.;.1, p.233-240, 1996.

\section{cross ref}

PORTER, M. E. Vantagem Competitiva: criando e sustentando um desempenho superior, $7^{\mathrm{a}}$ ed., Campus, RJ., 1992, 512p.

RUI, C.; RECHE, R. A.; BIZOTTO, B. L. S.; LAZARROTTO, E.; COSTA, C. A.; CAMARGO, M. A. Estudo de caso dos impactos da crise global nos critérios competitivos estratégicos de empresas. Revista Gestão Industrial, v. 7, n. 1, p. 23-42, 2011.

cross ref

SANTOS, S. R. Analise Crítica de um Método para Implantação das Normas ISO 9000 em Pequenas e Médias Empresas Brasileiras. Programa de Pós-Graduação em Engenharia Mecânica - Universidade Estadual de Campinas. Campinas, 2006.

SHARP, J. Guide to Good Pharmaceutical Manufacturing Practice. Guide to Good Pharmaceutical Manufacturing Practice, third edition, 1983, London, HMSO.

SO SERVIÇOS ORGANIZACIONAIS LTDA. O que é a RDC 59 da ANVISA? Disponível em: <http://www.rdc59.com.br/>. Acesso em: Janeiro 2011.

TROSCHINETZ, A. ISO 13485: Medical Devices and Risk Management. Quality, v. 49, 2010.

VAZ, C. R; TASCA, J. E; ENSSLIN, L.; ENSSLIN, S. R.; SELIG, P. M. Avaliação de desempenho na gestão estratégica organizacional: seleção de um referencial teórico de pesquisa e análise bibliométrica. Revista Gestão Industrial, v.8, n.4, p. 121-153, 2012.

WILLIG, S. H. Good manufacturing practices for pharmaceuticals: a plan for total quality control from manufacturer to consumer (5th edition, revised and enlarged). International Journal of Pharmaceutics, v. 218, n.1-2, p. 165-166, 2001.

\section{Dados dos autores:}

\section{Nome completo: Eduardo Gadotti Martins}

Filiação institucional: PUCPR

Departamento: Programa de Pós-Graduação em Engenharia de Produção e Sistemas

Função ou cargo ocupado: Mestrando

Endereço completo para correspondência: Rua Bassamo de Grappa, 49 - Sob M. Bairro Santa

Felicidade - Curitiba PR

Telefones para contato: (41) 99502688 
e-mail: eduardo.gadotti@pucpr.br; edugadotti@gmail.com

Nome completo: Edson Pinheiro de Lima

Filiação institucional: PUCPR

Departamento: Programa de Pós-Graduação em Engenharia de Produção e Sistemas

Função ou cargo ocupado: Professor Titular

Endereço completo para correspondência (bairro, cidade, estado, país e CEP): Rua Imaculada

Conceição, 1155 - Prado Velho - 80215901 - Curitiba, PR - Brasil

Telefones para contato: (41) 32711333 Fax: (41) 32711345

e-mail: e.pinheiro@pucpr.br

Enviado em: 04/12/2011

Aprovado em: 14/02/2013 